MATHEMATICS OF COMPUTATION

Volume 72 , Number 241, Pages 1-15

S $0025-5718(01) 01406-5$

Article electronically published on December 5, 2001

\title{
OVERLAPPING SCHWARZ PRECONDITIONERS FOR INDEFINITE TIME HARMONIC MAXWELL EQUATIONS
}

\author{
JAYADEEP GOPALAKRISHNAN AND JOSEPH E. PASCIAK
}

\begin{abstract}
Time harmonic Maxwell equations in lossless media lead to a second order differential equation for the electric field involving a differential operator that is neither elliptic nor definite. A Galerkin method using Nedelec spaces can be employed to get approximate solutions numerically. The problem of preconditioning the indefinite matrix arising from this method is discussed here. Specifically, two overlapping Schwarz methods will be shown to yield uniform preconditioners.
\end{abstract}

\section{INTRODUCTION}

Finite element methods for numerical solution of time harmonic Maxwell equations are now well established [4, 20, 21]. A class of these methods are Galerkin methods based on variational equations for the electric field involving a bilinear form in $\mathbf{H}_{0}(\operatorname{curl} ; \Omega)=\left\{\mathbf{u} \in\left(L^{2}(\Omega)\right)^{3}: \operatorname{curl} \mathbf{u} \in\left(L^{2}(\Omega)\right)^{3}, \mathbf{n} \times \mathbf{u}=0\right.$ on $\left.\partial \Omega\right\}$. Here $\Omega$ is a polyhedral open simply connected subset of $\mathbb{R}^{3}$, and $\mathbf{n}$ denotes the outward unit normal on the boundary $\partial \Omega$. The bilinear form is coercive in $\mathbf{H}_{0}(\mathbf{c u r l} ; \Omega)$ if the medium occupying $\Omega$ has positive electric conductivity. In this case, the linear system arising from the Galerkin method can be preconditioned using a preconditioner for the inner product on $\mathbf{H}_{0}(\mathbf{c u r l} ; \Omega)$. Such preconditioners can be constructed using the well known overlapping Schwarz method [8, 9, 10, 18] as shown in [16, 25, 26]. In this paper we analyze two overlapping Schwarz methods applied to the case when electric conductivity is zero, i.e., the case of undamped propagation in lossless media. In this case, the resulting linear system is indefinite.

In lossless media, the second order differential equation for the electric field that Maxwell equations yield [17] is not elliptic and is indefinite. These two difficulties complicate the analysis of the finite element method. Error estimates have been proved provided that the mesh size is sufficiently small [20].

These difficulties also complicate the analysis of possible preconditioners. Some recent analyses have successfully overcome the difficulties caused by the nonellipticity by means of a Helmholtz decomposition [2, 15, 16]. Our analysis will make use of some of the ideas in these works. We must also handle the difficulties arising from the indefiniteness of the system. Some suggestions for overcoming this difficulty appear in [22], wherein a regularizing term is added to make the system positive

Received by the editor July 10, 2000 and, in revised form, March 7, 2001.

2000 Mathematics Subject Classification. Primary 65F10, 65N55, 65N30.

Key words and phrases. Schwarz method, indefinite, Maxwell equations, preconditioner, domain decomposition, finite element.

The first author was supported in part by Medtronic Inc.

The second author was partially supported by NSF grant number DMS-9973328. 
definite on potential fields. In contrast, we will analyze the system as it is. This is in the spirit of the perturbation approach used in [6] for analyzing Schwarz methods for indefinite elliptic problems. Moreover, our perturbation arguments can be used to analyze multigrid methods as well [14].

We restrict our attention to time harmonic Maxwell equations in a homogeneous lossless medium occupying $\Omega$. We also assume that the boundary of $\Omega$ is adjacent to a perfect conductor. For the sake of simplicity of presentation, we set material properties (magnetic permeability and electric permittivity) equal to unity. Maxwell equations then gives rise to the following variational formulation [7, 20] for the electric field $\mathbf{U} \in \mathbf{H}_{0}(\operatorname{curl} ; \Omega)$ :

$$
\mathbf{A}(\mathbf{U}, \mathbf{v})=(\mathbf{J}, \mathbf{v}) \quad \text { for all } \mathbf{v} \in \mathbf{H}_{0}(\operatorname{curl} ; \Omega),
$$

where

$$
\mathbf{A}(\mathbf{U}, \mathbf{v})=(\operatorname{curl} \mathbf{U}, \operatorname{curl} \mathbf{v})-\omega^{2}(\mathbf{U}, \mathbf{v})
$$

and $(\cdot, \cdot)$ denotes the $\left(L^{2}(\Omega)\right)^{3}$ inner product. The vector $\mathbf{J}$ is proportional to the electric current and satisfies $\operatorname{div} \mathbf{J}=0$, consequently $\operatorname{div} \mathbf{U}=0$. In (1.2), $\omega$ is a real number denoting frequency of propagation. Note that there is a countable set of real values for $\omega$ for which (1.1) does not have a unique solution [17]. Throughout this paper we assume that $\omega$ is not one of these values and so (1.1) is uniquely solvable.

In our arguments later, we will need to assume that solutions to (1.1) are regular. Let $\Omega$ be convex. It is well known ([20, cf. [12]) that there is a constant $C_{\Omega}$ depending only on $\Omega$ such that

$$
\|\mathbf{U}\|_{H^{1}}+\|\operatorname{curl} \mathbf{U}\|_{H^{1}} \leq C_{\Omega}\|\mathbf{J}\| .
$$

In (1.3), $\|\cdot\|_{H^{1}}$ denotes the norm of $\left(H^{1}(\Omega)\right)^{3}$ and $H^{1}(\Omega)=\left\{u \in L^{2}(\Omega): \operatorname{grad} u \in\right.$ $\left.\left(L^{2}(\Omega)\right)^{3}\right\}$. We use $\|\cdot\|$ to denote the norm in $\left(L^{2}(\Omega)\right)^{3}$. For later use, let us also set

$$
\begin{aligned}
H_{0}^{1}(\Omega) & =\left\{u \in H^{1}(\Omega): u=0 \text { on } \partial \Omega\right\}, \\
\mathbf{H}_{0}(\operatorname{div} ; \Omega) & =\left\{\mathbf{u} \in\left(L^{2}(\Omega)\right)^{3}: \operatorname{div} \mathbf{u} \in L^{2}(\Omega) \text { and } \mathbf{u} \cdot \mathbf{n}=0 \text { on } \partial \Omega\right\} .
\end{aligned}
$$

In the notation for function spaces and their norms and inner products, when the domain is absent, it is to be taken as $\Omega$; e.g., $\mathbf{H}_{0}(\mathbf{c u r l}) \equiv \mathbf{H}_{0}(\mathbf{c u r l} ; \Omega)$.

\section{Discrete SPACES}

The overlapping Schwarz algorithm as described in [8, 9, 10, 24 is based on two levels of partitioning of $\Omega$. The first is a coarse partitioning into (nonoverlapping) tetrahedra $\left\{\Omega_{i}: i=1, \ldots N\right\}$. This forms a quasiuniform mesh of mesh-size $H$. Next, each $\Omega_{i}$ is further partitioned into finer tetrahedra $\left\{\tau_{i}^{j}: j=1,2 \ldots\right\}$. These partitionings are such that taken together they form a quasiuniform mesh of $\Omega$ of mesh size $h$. Let $\mathbf{Q}_{h}$ and $\mathbf{Q}_{H}$ denote Nedelec finite element subspaces [21] of $\mathbf{H}_{0}(\mathbf{c u r l})$ of index $k$ based on meshes $\left\{\tau_{i}^{j}\right\}$ and $\left\{\Omega_{i}\right\}$, respectively. A function in $\mathbf{Q}_{H}$, for example, is of the form $\mathbf{p}(\mathbf{x})+\mathbf{r}(\mathbf{x})$ when $\mathbf{x}$ is restricted to a tetrahedron $\Omega_{i}$, where $\mathbf{p}$ and $\mathbf{r}$ are such that each of their three components are polynomials of degree at most $k$ and $k+1$, respectively, and $\mathbf{r} \cdot \mathbf{x}=0$. Our results hold if Nedelec edge elements based on cubes are used instead of tetrahedral elements. 


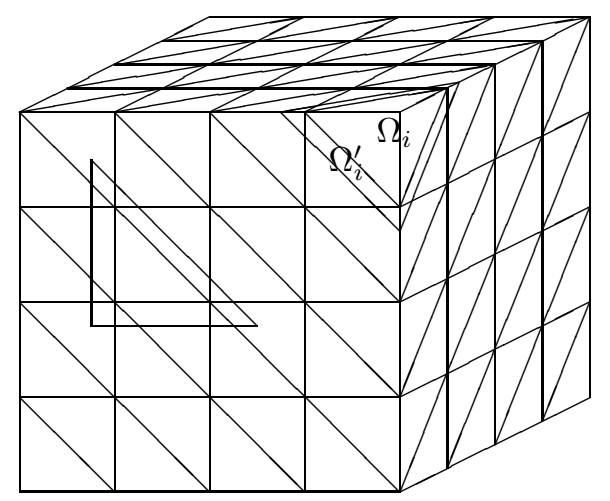

Figure 1. An example of domain decomposition

The discrete approximation $\mathbf{U}_{h} \in \mathbf{Q}_{h}$ is defined by

$$
\mathbf{A}\left(\mathbf{U}_{h}, \mathbf{v}\right)=(\mathbf{J}, \mathbf{v}) \text { for all } \mathbf{v} \in \mathbf{Q}_{h} .
$$

It is proved in [20] that $\mathbf{U}_{h}$ exists uniquely whenever $h$ is sufficiently small. Using (1.3), and the arguments in [20], it is easy to see that there exists an $\bar{h}_{0}>0$ such that whenever $h \leq \bar{h}_{0}$,

$$
\left\|\mathbf{U}-\mathbf{U}_{h}\right\|_{\boldsymbol{\Lambda}} \leq C h\|\mathbf{J}\| .
$$

Here $\|\cdot\|_{\boldsymbol{\Lambda}}$ denotes the norm in $\mathbf{H}_{0}(\mathbf{c u r l})$, i.e., $\|\mathbf{u}\|_{\boldsymbol{\Lambda}}=\boldsymbol{\Lambda}(\mathbf{u}, \mathbf{u})^{1 / 2}$ with

$$
\mathbf{\Lambda}(\mathbf{u}, \mathbf{v})=(\operatorname{curl} \mathbf{u}, \operatorname{curl} \mathbf{v})+(\mathbf{u}, \mathbf{v}) \text {. }
$$

Throughout this paper $C$, with or without subscripts, denotes a generic constant independent of $h$ and $H$. Its value may differ at different occurrences.

Let us recall some well known relationships between Nedelec spaces and two other discrete spaces. Let $\mathbf{V}_{h}$ denote Raviart-Thomas finite element subspaces [5] of $\mathbf{H}_{0}$ (div) of index $k$ based on the $h$-level mesh. A function in $\mathbf{V}_{h}$ is of the form $\mathbf{p}(\mathbf{x})+r(\mathbf{x}) \mathbf{x}$ when $\mathbf{x}$ is restricted to a fine tetrahedron, where all three components of $\mathbf{p}$ as well as $r$ are polynomials of degree at most $k$. Let $W_{h}$ denote the subspace of $H_{0}^{1}(\Omega)$ consisting of functions which when restricted to a fine tetrahedron are polynomials of degree at most $k+1$. We will repeatedly use the following well known [2, 15, 16] orthogonal decomposition of $\mathbf{Q}_{h}$ of Helmholtz type:

$$
\mathbf{Q}_{h}=\operatorname{curl}_{h} \mathbf{V}_{h} \oplus \operatorname{grad} W_{h} .
$$

Here $\operatorname{curl}_{h}$ is the $L^{2}$-adjoint of the map curl $: \mathbf{Q}_{h} \rightarrow \mathbf{V}_{h}$. Our notation is close to that in 2]. Since we have assumed that $\Omega$ is convex, it follows from [13] that the "Poincaré inequality",

$$
\left\|\mathbf{q}_{h}\right\| \leq C\left\|\operatorname{curl} \mathbf{q}_{h}\right\|
$$

holds for all $\mathbf{q}_{h} \in \operatorname{curl}_{h} \mathbf{V}_{h}$.

We now introduce notation for overlapping subregions and associated spaces. Extend each $\Omega_{i}$ to a larger region $\Omega_{i}^{\prime}$ (i.e., $\Omega_{i} \subset \Omega_{i}^{\prime} \subset \Omega$, see Figure 1), in such a way that $\partial \Omega_{i}^{\prime}$ aligns with the $h$-level mesh. Then each $\Omega_{i}^{\prime}$ is also partitioned by a subset of $\left\{\tau_{i}^{j}\right\}_{i, j}$, and the space

$$
\mathbf{Q}^{i}=\mathbf{Q}_{h} \cap \mathbf{H}_{0}\left(\operatorname{curl} ; \Omega_{i}^{\prime}\right), \quad(i=1, \ldots N)
$$


is again a Nedelec finite element space. In the above definition, we consider $\mathbf{H}_{0}\left(\mathbf{c u r l} ; \Omega_{i}^{\prime}\right)$ as a subset of $\mathbf{H}_{0}(\mathbf{c u r l})$ by identifying functions in $\mathbf{H}_{0}\left(\mathbf{c u r l} ; \Omega_{i}^{\prime}\right)$ with their extension by zero. We assume that each $\Omega_{i}^{\prime}$ is convex and that the collection $\left\{\Omega_{i}^{\prime}: i=1, \ldots N\right\}$ satisfies the following conditions:

1. Generous overlap: There is a constant $\delta>0$ such that

$$
\operatorname{dist}\left(\partial \Omega_{i}^{\prime} \cap \Omega, \partial \Omega_{i} \cap \Omega\right) \geq \delta H \quad \text { for all } i=1, \ldots N .
$$

2. Finite covering: Every point of $\Omega$ belongs to at most $\rho$ subdomains in $\left\{\Omega_{i}^{\prime}\right.$ : $i=1, \ldots N\}$.

3. $H$-independent uniformity: There are a fixed number of reference domains $\left\{\widehat{\Omega}_{i}\right\}$ such that each subdomain $\Omega_{j}^{\prime}$, for $j=1, \ldots, N$, is the image under a linear transformation $F_{j}(\hat{x})=B_{j} \hat{x}+b_{j}$ of one of the reference domains, $\widehat{\Omega}_{i}$. We assume that the matrix $B_{j}$ is a multiple $\alpha_{j}$ times a unitary matrix $U_{j}$ and that $\alpha_{j}$ satisfies

$$
C_{0} H \leq\left|\alpha_{j}\right| \leq C_{1} H
$$

The generic constants $C$ in our estimates will be allowed to depend on $\delta, \rho$, and the geometric properties of the reference domains $\left\{\widehat{\Omega}_{i}\right\}$. For convenient notation let us also set $\mathbf{Q}^{0}=\mathbf{Q}_{H}$, and $\Omega_{0}^{\prime}=\Omega$.

Remark 2.1. The first two conditions above are standard in papers analyzing Schwarz algorithms. A condition such as the third, although necessary for proving estimates independent of $H$ in many of the analyses, is seldom stated. For example, in the analysis of overlapping methods for elliptic second order problems, it seems necessary to assume that the Lipschitz constants for the overlapping subdomains can be bounded independently of $H$.

Remark 2.2. We make the assumption that subdomains $\Omega_{j}^{\prime}$ are convex only so that we can invoke Proposition 5.1 in Chapter III of 13 to obtain the Poincaré inequality (2.4) for discretely divergence free functions on the (convex) reference domains $\widehat{\Omega}_{i}$ (see (4.3) in particular). Recent results indicate that such an inequality holds under weaker assumptions [1, 19. Our analysis remains unchanged if these assumptions are made instead of convexity.

Now define $\mathbf{V}^{0}$ and $W^{0}$ in the same way as $\mathbf{V}_{h}$ and $W_{h}$ but using the $H$-level mesh. Also set

$$
\mathbf{V}^{i}=\mathbf{V}_{h} \cap \mathbf{H}_{0}\left(\operatorname{div} ; \Omega_{i}^{\prime}\right) \text { and } W^{i}=W_{h} \cap H_{0}^{1}\left(\Omega_{i}^{\prime}\right),
$$

for all $i=1, \ldots, N$. Here, again, we implicitly extend (by zero) functions on $\Omega^{\prime}$ to $\Omega$. Decompositions analogous to (2.3) hold for $\mathbf{Q}^{i}$ in terms of $\mathbf{V}^{i}$ and $W^{i}$, i.e.,

$$
\mathbf{Q}^{i}=\operatorname{curl}_{h}^{i} \mathbf{V}^{i} \oplus \operatorname{grad} W^{i} .
$$

Here $\operatorname{curl}_{h}^{i}$ is the $L^{2}$-adjoint of the map curl $: \mathbf{Q}^{i} \rightarrow \mathbf{V}^{i}$.

\section{The overlapping Schwarz Preconditioner}

In this section we give two overlapping Schwarz preconditioners for the discrete systems corresponding to (2.1). Specifically, we consider an additive Schwarz preconditioner and a two-level multiplicative preconditioner. We also give the main 
theorems of this paper, which can be used to guarantee that appropriate iterative schemes converge with rates that are bounded independently of the mesh and subdomain parameters. Their proofs will appear in the next section.

These preconditioners are based on inversions on the overlapping subregions $\left\{\Omega_{i}^{\prime}\right\}$. In our subsequent analysis, we will show that the problem of finding a $\mathbf{v}_{i} \in \mathbf{Q}^{i}$ satisfying

$$
\mathbf{A}\left(\mathbf{v}_{i}, \mathbf{w}_{i}\right)=\ell\left(\mathbf{w}_{i}\right) \quad \text { for all } \mathbf{w}_{i} \in \mathbf{Q}^{i},
$$

is uniquely solvable for $i=0, \ldots, N$ provided that $H$ is sufficiently small. Here $\ell$ is a linear functional on $\mathbf{Q}^{i}$. We will always assume that $H$ is so small that (3.1) is uniquely solvable.

Let $\left\{\boldsymbol{\phi}_{i}\right\}$ denote the nodal basis for $\mathbf{Q}_{h}$. In implementation, the solution of (2.1) is reduced to a matrix problem of the form

$$
A x=b,
$$

where $A_{i j}=\mathbf{A}\left(\phi_{j}, \phi_{i}\right)$ is the stiffness matrix, $x$ is the coefficient vector for the solution $\mathbf{U}_{h}$ and $b_{i}=\left(\mathbf{J}, \phi_{i}\right)$. The matrix $A$ can be thought of as the matrix of a linear map (which we also denote by $\mathbf{A}$ ) from $\mathbf{Q}_{h}$ to its dual, $\left(\mathbf{Q}_{h}\right)^{\prime}$. Indeed, a functional in the dual space is represented by its values applied to the nodal basis functions. Thus, $(A v)_{j}=\sum_{i} A_{i j} v_{i}=\mathbf{A}\left(\mathbf{v}, \phi_{j}\right)$, where $\mathbf{v}$ is the function in $\mathbf{Q}_{h}$ corresponding to $v$, i.e., $A v$ corresponds to the functional $(\mathbf{A v})(\cdot)=\mathbf{A}(\mathbf{v}, \cdot)$. A preconditioning matrix should therefore correspond to a map from $\left(\mathbf{Q}_{h}\right)^{\prime}$ back to $\mathbf{Q}_{h}$.

We first define the additive Schwarz preconditioner, namely $\mathbf{B}_{a}:\left(\mathbf{Q}_{h}\right)^{\prime} \rightarrow \mathbf{Q}_{h}$. For $\ell \in\left(\mathbf{Q}_{h}\right)^{\prime}$, set

$$
\mathbf{B}_{a}(\ell)=\sum_{i=0}^{N} \mathbf{v}_{i},
$$

where for each $i, \mathbf{v}_{i}$ is the solution of the problem

$$
\mathbf{A}\left(\mathbf{v}_{i}, \mathbf{w}_{i}\right)=\ell\left(\mathbf{w}_{i}\right) \quad \text { for all } \mathbf{w}_{i} \in \mathbf{Q}^{i} .
$$

We let the corresponding matrix be denoted by $B_{a}$. Computing the product of $B_{a}$ with the vector representing $\ell$ involves finding nodal coefficients of the $\mathbf{v}_{i}$ in (3.3). For the case $i=0$, this involves a change of basis from the coarse grid basis for $\mathbf{Q}^{0}$ and the computation of $\ell$ applied to coarse grid basis functions. These two operations are often called "prolongation" and "restriction", respectively.

It is immediate from the above definitions that $\mathbf{B}_{a} \mathbf{A}=\sum_{i=0}^{N} \mathbf{T}_{i}$, where $\mathbf{T}_{i} \mathbf{w}=$ $\mathbf{v}_{i} \in \mathbf{Q}^{i}$ solves the problem

$$
\mathbf{A}\left(\mathbf{v}_{i}, \phi\right)=\mathbf{A}(\mathbf{w}, \phi) \quad \text { for all } \phi \in \mathbf{Q}^{i} .
$$

The following theorem bounds the spectrum of $\mathbf{B}_{a} \mathbf{A}$.

Theorem 3.1. There exists $\bar{h}_{1}>0$ such that for all $H \leq \bar{h}_{1}$, and for all $\mathbf{u} \in \mathbf{Q}_{h}$,

$$
\begin{aligned}
c_{1} \boldsymbol{\Lambda}(\mathbf{u}, \mathbf{u}) & \leq \boldsymbol{\Lambda}\left(\mathbf{B}_{a} \mathbf{A u}, \mathbf{u}\right), \\
\boldsymbol{\Lambda}\left(\mathbf{B}_{a} \mathbf{A u}, \mathbf{v}\right) & \leq c_{2} \boldsymbol{\Lambda}(\mathbf{u}, \mathbf{u})^{1 / 2} \boldsymbol{\Lambda}(\mathbf{v}, \mathbf{v})^{1 / 2} .
\end{aligned}
$$

Here $c_{1}$ and $c_{2}$ are constants independent of $h$ and $H$. 
The above result can be used to guarantee convergence rates for Krylov space iterative methods such as GMRES [11, 23]. For example, we have the following corollary:

Corollary 3.1. Let $\mathbf{v}_{i}, i=1,2, \ldots$, be the sequence of iterates obtained when GMRES in the $\mathbf{\Lambda}(\cdot, \cdot)$-innerproduct is applied to the preconditioned system $\mathbf{B}_{a} \mathbf{A v}=$ $\mathbf{B}_{a} \ell$, with $\mathbf{v}_{0}$ as initial iterate. If $H \leq \bar{h}_{0}$, then

$$
\left\|\mathbf{v}-\mathbf{v}_{i}\right\|_{\Lambda}^{2} \leq \eta^{i}\left\|\mathbf{v}-\mathbf{v}_{0}\right\|_{\Lambda}^{2}
$$

with $\eta=1-c_{1}^{2} / c_{2}^{2}$.

Remark 3.1. The above corollary implies that GMRES applied to the preconditioned matrix problem

$$
B_{a} A u=B_{a} b
$$

in the inner product induced by the matrix $\left\{\Lambda_{i j}\right\}=\left\{\boldsymbol{\Lambda}\left(\boldsymbol{\phi}_{i}, \boldsymbol{\phi}_{j}\right)\right\}$ converges with a reduction rate of $\eta$ per step.

Remark 3.2. In the case of elliptic second order indefinite problem, replacing the subdomain inversions by uniform preconditioners gives rise to a uniform global preconditioner [6]. This apparently is not the case in our application. Indeed, we give numerical results in Section 5 which suggest that the additive Schwarz preconditioner with subdomain inversions replaced by certain positive definite subdomain solutions operators performs rather poorly. Note that in the elliptic case, if the associated bilinear form satisfies a Gårding inequality, it becomes coercive on subdomain spaces for sufficiently small subdomain sizes. It is therefore natural to replace the subdomain inversions by other (more efficient) positive definite preconditioners. In our application however, subdomain inversions are never positive definite.

The second algorithm which we will consider is a two-level multiplicative algorithm. Let $\alpha>0$ be a scaling parameter to be chosen later. For any $\ell \in\left(\mathbf{Q}_{h}\right)^{\prime}$, the action of the two-level operator on $\ell$, namely $\mathbf{B}_{m}(\ell)$, is defined as follows:

1. Let $\mathbf{v}_{0}$ be defined by (3.3).

2. For $i=1, \ldots, N$, define $\mathbf{v}_{i} \in \mathbf{Q}^{i}$ by

$$
\mathbf{A}\left(\mathbf{v}_{i}, \mathbf{w}_{i}\right)=\ell\left(\mathbf{w}_{i}\right)-\mathbf{A}\left(\mathbf{v}_{0}, \mathbf{w}_{i}\right) \quad \text { for all } \mathbf{w}_{i} \in \mathbf{Q}^{i} .
$$

3. Set $\mathbf{B}_{m}(\ell)=\mathbf{v}_{0}+\alpha \sum_{i=1}^{N} \mathbf{v}_{i}$.

A standard computation shows that

$$
\mathbf{I}-\mathbf{B}_{m} \mathbf{A}=(\mathbf{I}-\alpha \widetilde{\mathbf{T}})\left(\mathbf{I}-\mathbf{T}_{0}\right)
$$

where $\mathbf{I}$ denotes the identity operator, and $\widetilde{\mathbf{T}}$ is given by

$$
\widetilde{\mathbf{T}}=\sum_{i=1}^{N} \mathbf{T}_{i} .
$$

A convergence result for this product method is given by the following theorem.

Theorem 3.2. There exists an $\alpha_{0}>0$ such that, for all $\alpha \leq \alpha_{0}$, there is an $\bar{h}_{2}>0$ (depending on $\alpha$ ) such that, whenever $H \leq \bar{h}_{2}$,

$$
\left\|\left(\mathbf{I}-\mathbf{B}_{m} \mathbf{A}\right) \mathbf{u}\right\|_{\boldsymbol{\Lambda}} \leq \gamma\|\mathbf{u}\|_{\boldsymbol{\Lambda}}
$$

for all $\mathbf{u} \in \mathbf{Q}_{h}$ with $\gamma<1$ independent of $h$ and $H$. 


\section{AnAlysis}

In this section, we provide an analysis of the two domain decomposition algorithms given in the previous section. The proofs of the main theorems are given at the end of this section after we develop a sequence of auxiliary results.

Let us first establish that when $H$ is small enough, there is a unique solution to (3.1). The case of $i=0$ follows directly from [20]. For $i>0$, we require the Poincaré inequality given in the following lemma.

Lemma 4.1. There is a constant $c_{0}$, independent of $h$ and $H$, such that, for $i=$ $1, \ldots, N$,

$$
\|\mathbf{q}\|_{0, \Omega_{i}^{\prime}} \leq c_{0} H\|\mathbf{c u r l} \mathbf{q}\|_{0, \Omega_{i}^{\prime}} \text { for all } \mathbf{q} \in \operatorname{curl}_{h}^{i} \mathbf{V}^{i}
$$

Here $\|\cdot\|_{0, \Omega_{i}^{\prime}}$ denotes the $\left(L^{2}\left(\Omega_{i}^{\prime}\right)\right)^{3}-$ norm.

Proof. Fix $\Omega_{j}^{\prime}$ and let $F_{j}$ be the linear transformation given by our $H$-independent uniformity assumption on $\left\{\Omega_{j}^{\prime}\right\}$. For a vector function $\mathbf{u}$ defined on $\Omega_{j}^{\prime}$ let $\widehat{\mathbf{u}}$ be the vector function defined on the reference domain $\widehat{\Omega}_{i}$ given by

$$
\widehat{\mathbf{u}}=B_{j}^{t}\left(\mathbf{u} \circ F_{j}\right) .
$$

Clearly, $\widehat{\Omega}_{i}$ inherits a finite element mesh from $\Omega_{j}^{\prime}$, and we denote the corresponding approximation spaces $\widehat{W}^{j}$ and $\widehat{\mathbf{Q}}^{j}$. Moreover, the map $\mathbf{u} \mapsto \widehat{\mathbf{u}}$ is a bijection of $\mathbf{Q}^{j}$ onto $\widehat{\mathbf{Q}}^{j}[21$. Since the scalar transformation

$$
\widehat{\phi}=\phi \circ F_{j}
$$

maps $W^{j}$ bijectively onto $\widehat{W}^{j}$, a change of variable shows that $\mathbf{q} \in \operatorname{curl}_{h}^{j} \mathbf{V}^{j}$ if and only if

$$
(\widehat{\mathbf{q}}, \operatorname{grad} \widehat{\phi})_{\widehat{\Omega}_{i}}=\left|\operatorname{det} B_{j}^{-1}\right|\left(B_{j}^{t} \mathbf{q}, B_{j}^{t} \operatorname{grad} \phi\right)_{\Omega_{j}^{\prime}}=0 \quad \text { for all } \phi \in \widehat{W}^{j}
$$

Here $(\cdot, \cdot) \widehat{\Omega}_{i}$ denotes the inner product in $\left(L^{2}\left(\widehat{\Omega}_{i}\right)\right)^{3}$. The Poincaré inequality holds for $\widehat{\mathbf{q}}$ satisfying (4.2) (see, e.g., [13]), i.e.,

$$
\|\widehat{\mathbf{q}}\|_{\widehat{\Omega}_{i}} \leq C\|\operatorname{curl} \widehat{\mathbf{q}}\|_{\widehat{\Omega}_{i}} .
$$

Let $q_{l}$ and $\widehat{q}_{l}$ for $l=1,2,3$ denote the components of $\mathbf{q}$ and $\widehat{\mathbf{q}}$, respectively, and consider the matrices

$$
\begin{aligned}
& M_{i j}=\left(\partial q_{j} / \partial x_{i}-\partial q_{i} / \partial x_{j}\right), \\
& \widehat{M}_{i j}=\left(\partial \widehat{q}_{j} / \partial \widehat{x}_{i}-\partial \widehat{q}_{i} / \partial \widehat{x}_{j}\right)
\end{aligned}
$$

A straightforward computation gives that

$$
\widehat{M}=B_{j}^{t}\left(M \circ F_{j}\right) B_{j}
$$

This implies that

$$
|\operatorname{curl} \widehat{\mathbf{q}}(\widehat{x})|=\alpha_{i}^{2}|\operatorname{curl} \mathbf{q}(x)| \text { for all } x \in \Omega_{j}^{\prime} .
$$

The lemma follows from (2.6), (4.3), (4.4) and a change of integration variable.

Remark 4.1. The $H$-independent uniformity assumption was only made so that we could prove (4.1). Our results will hold without this assumption if one somehow has (4.1). One might expect that (4.1) would fail to hold if the subdomains were generated by some automatic mesh partitioning algorithms. 
We can now prove the unique solvability of (3.1) as asserted in the following lemma.

Lemma 4.2. There exists an $\bar{h}_{3}>0$ such that whenever $H \leq \bar{h}_{3}$, any solution $\mathbf{t}_{i}$ of

$$
\mathbf{A}\left(\mathbf{t}_{i}, \mathbf{v}_{i}\right)=\mathbf{A}\left(\mathbf{u}, \mathbf{v}_{i}\right) \quad \text { for all } \mathbf{v}_{i} \in \mathbf{Q}^{i}
$$

satisfies

$$
\left\|\mathbf{t}_{i}\right\|_{\boldsymbol{\Lambda}, \Omega_{i}^{\prime}} \leq C\|\mathbf{u}\|_{\boldsymbol{\Lambda}, \Omega_{i}^{\prime}} \quad(i=1, \ldots, N)
$$

for $\mathbf{u} \in \mathbf{Q}_{h}$. In particular, this implies that for $i \geq 1$, 3.1) has a unique solution when $H \leq \bar{h}_{3}$.

Proof. Let $\mathbf{t}_{i}$ be a solution of (4.5). Using the decomposition (2.3), we first write

$$
\mathbf{t}_{i}=\operatorname{grad} \phi_{i}+\operatorname{curl}_{h}^{i} \mathbf{w}_{i}, \quad \phi_{i} \in W^{i}, \mathbf{w}_{i} \in \mathbf{V}^{i} .
$$

Then, by the definition of $\mathbf{t}_{i}$,

$$
\left(\operatorname{grad} \phi_{i}, \operatorname{grad} \psi_{i}\right)_{\Omega_{i}^{\prime}}=\left(\mathbf{u}, \operatorname{grad} \psi_{i}\right)_{\Omega_{i}^{\prime}}
$$

for all $\psi_{i} \in W^{i}$. This implies that

$$
\left\|\operatorname{grad} \phi_{i}\right\|_{0, \Omega_{i}^{\prime}} \leq\|\mathbf{u}\|_{0, \Omega_{i}^{\prime}}
$$

The remainder, $\operatorname{curl}_{h}^{i} \mathbf{w}_{i}$, satisfies

$$
\begin{aligned}
& \left\|\operatorname{curl} \operatorname{curl}_{h} \mathbf{w}_{i}\right\|_{0, \Omega_{i}^{\prime}}^{2}-\omega^{2}\left\|\operatorname{curl}_{h} \mathbf{w}_{i}\right\|_{0, \Omega_{i}^{\prime}}^{2} \\
& =\left(\operatorname{curl} \mathbf{u}, \operatorname{curl} \operatorname{curl}_{h} \mathbf{w}_{i}\right)_{\Omega_{i}^{\prime}}-\omega^{2}\left(\mathbf{u}, \operatorname{curl}_{h} \mathbf{w}_{i}\right)_{\Omega_{i}^{\prime}} .
\end{aligned}
$$

By (4.1),

$$
\left\|\operatorname{curl}_{h} \mathbf{w}_{i}\right\|_{0, \Omega_{i}^{\prime}} \leq c_{0} H\left\|\operatorname{curl} \operatorname{curl}_{h} \mathbf{w}_{i}\right\|_{0, \Omega_{i}^{\prime}} .
$$

Thus

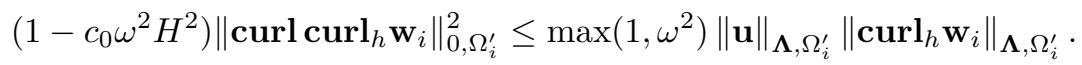

The above two inequalities imply that

$$
\left\|\operatorname{curl}_{h} \mathbf{w}_{i}\right\|_{\Lambda, \Omega_{i}^{\prime}} \leq C\|\mathbf{u}\|_{\Lambda, \Omega_{i}^{\prime}},
$$

for all $H \leq \bar{h}_{3}$, if $\bar{h}_{3}$ is chosen such that $\left(1-c_{0} \omega^{2} \bar{h}_{3}^{2}\right)>0$. Combining the above results proves (4.6).

Finally, (3.1) is uniquely solvable if and only if the stiffness matrix $A$ for $\mathbf{A}(\cdot, \cdot)$ is nonsingular. However, (4.6) implies $A$ is one to one. This completes the proof of the lemma.

Before providing our next perturbation lemma, we require some additional machinery. For $\mathbf{q}_{h} \in \mathbf{c u r l}_{h} \mathbf{V}_{h}$, define $\mathbf{S q}_{h}$ to be the unique function $\mathbf{a} \in \mathbf{H}_{0}(\mathbf{c u r l})$ satisfying

$$
\operatorname{curl} \mathbf{a}=\operatorname{curl} \mathbf{q}_{h}, \quad \operatorname{div} \mathbf{a}=0 .
$$

It can be shown [2] that $\mathbf{S}$ is well defined and satisfies

$$
\left\|\mathbf{q}_{h}-\mathbf{S q}_{h}\right\| \leq C h\left\|\operatorname{curl} \mathbf{q}_{h}\right\| \text {. }
$$

Thus, although $\mathbf{q}_{h}$ is not solenoidal, $\mathbf{S} \mathbf{q}_{h}$ provides an approximation to $\mathbf{q}_{h}$ that is solenoidal and satisfies $\operatorname{curl} \mathbf{S} \mathbf{q}_{h}=\operatorname{curl} \mathbf{q}_{h}$. 
Our next result estimates the components of $\mathbf{u}-\mathbf{T}_{i} \mathbf{u}$ along the subspace $\mathbf{Q}^{i}$. This result is crucial for a perturbation argument used to prove the main theorems.

Lemma 4.3. There exists $\bar{h}_{4}>0$ such that, for any $i=0, \ldots, N$,

$$
\left(\mathbf{u}-\mathbf{T}_{i} \mathbf{u}, \mathbf{v}_{i}\right) \leq C H\left\|\mathbf{u}-\mathbf{T}_{i} \mathbf{u}\right\|_{\boldsymbol{\Lambda}, \Omega_{i}^{\prime}}\left\|\mathbf{v}_{i}\right\|_{\boldsymbol{\Lambda}, \Omega_{i}^{\prime}}
$$

for all $\mathbf{u} \in \mathbf{Q}_{h}$ and $\mathbf{v}_{i} \in \mathbf{Q}^{i}$ whenever $H \leq \bar{h}_{4}$.

Proof. Let $\mathbf{u}$ be in $\mathbf{Q}_{h}$ and $\mathbf{v}_{i}$ be in $\mathbf{Q}^{i}$. Let us use the decomposition in (2.3) and write

$$
\mathbf{e}_{h} \equiv \mathbf{u}-\mathbf{T}_{i} \mathbf{u}=\operatorname{grad} \phi_{h}+\operatorname{curl}_{h} \mathbf{r}_{h}, \quad \phi_{h} \in W_{h}, \mathbf{r}_{h} \in \mathbf{V}_{h}
$$

Observe that, by the definition of $\mathbf{T}_{i}$,

$$
\left(\mathbf{e}_{h}, \operatorname{grad} \phi^{i}\right)_{\Omega_{i}^{\prime}}=0 \text { for all } \phi \in W^{i} .
$$

We first consider $i=0$. Let $\mathbf{v}_{0} \in \mathbf{Q}^{0}$ be decomposed as

$$
\mathbf{v}_{0}=\operatorname{grad} \psi_{H}+\operatorname{curl}_{H} \mathbf{z}_{H}, \quad \psi_{H} \in W^{0}, \mathbf{z}_{H} \in \mathbf{V}^{0}
$$

By (4.11), we have

$$
\left(\mathbf{e}_{h}, \mathbf{v}_{0}\right)=\left(\operatorname{curl}_{h} \mathbf{r}_{h}, \operatorname{curl}_{H} \mathbf{z}_{H}\right)+\left(\operatorname{grad} \phi_{h}, \operatorname{curl}_{H} \mathbf{z}_{H}\right) .
$$

The two terms on right hand side will be estimated separately.

Consider the first term on the right hand side of (4.12). We will prove that

$$
\left\|\operatorname{curl}_{h} \mathbf{r}_{h}\right\| \leq C H\left\|\mathbf{e}_{h}\right\|_{\Lambda} .
$$

Set $\varepsilon=\mathbf{S}\left(\operatorname{curl}_{h} \mathbf{r}_{h}\right)$. From (4.10), it follows that

$$
\left\|\varepsilon-\operatorname{curl}_{h} \mathbf{r}_{h}\right\| \leq C h\left\|\mathbf{e}_{h}\right\|_{\Lambda} .
$$

Thus to verify (4.13), it suffices to estimate $\|\varepsilon\|$. We do this by a duality argument. Let $\mathbf{w} \in \mathbf{H}_{0}$ (curl) be defined by

$$
\mathbf{A}(\mathbf{w}, \mathbf{p})=(\varepsilon, \mathbf{p}) \quad \text { for all } \mathbf{p} \in \mathbf{H}_{0}(\mathbf{c u r l}) .
$$

Note that both $\varepsilon$ and $\mathbf{w}$ are divergence free. Since $\operatorname{curl} \varepsilon=\operatorname{curl}_{\mathbf{l}} \operatorname{curl}_{h} \mathbf{r}_{h}$, we have

$$
\|\varepsilon\|^{2}=\mathbf{A}(\mathbf{w}, \boldsymbol{\varepsilon})=\mathbf{A}\left(\mathbf{w}, \operatorname{curl}_{h} \mathbf{r}_{h}\right)+\omega^{2}\left(\operatorname{curl}_{h} \mathbf{r}_{h}-\varepsilon, \mathbf{w}\right)
$$

In the first term above, we can substitute $\mathbf{e}_{h}$ for $\mathbf{c u r l}_{h} \mathbf{r}_{h}$ since $\operatorname{div} \mathbf{w}=0$. Then, since $\mathbf{A}\left(\mathbf{w}_{H}, \mathbf{e}_{h}\right)=0$ for any $\mathbf{w}_{H} \in \mathbf{Q}_{H}$, we have

$$
\begin{aligned}
\|\varepsilon\|^{2} & =\mathbf{A}\left(\mathbf{w}-\mathbf{w}_{H}, \mathbf{e}_{h}\right)+\omega^{2}\left(\operatorname{curl}_{h} \mathbf{r}_{h}-\varepsilon, \mathbf{w}\right) \\
& \leq \max \left(1, \omega^{2}\right)\left\|\mathbf{w}-\mathbf{w}_{H}\right\|_{\boldsymbol{\Lambda}}\left\|\mathbf{e}_{h}\right\|_{\boldsymbol{\Lambda}}+\omega^{2}\left\|\mathbf{c u r l}_{h} \mathbf{r}_{h}-\varepsilon\right\|\|\mathbf{w}\| \\
& \leq C H\|\varepsilon\|\left\|\mathbf{e}_{h}\right\|_{\boldsymbol{\Lambda}}+C h\|\mathbf{w}\|\left\|\mathbf{e}_{h}\right\|_{\boldsymbol{\Lambda}} .
\end{aligned}
$$

To get the last inequality we used (2.2) and (4.14). Since $h \leq H$, and (1.3) holds for $\mathbf{w}$, it follows that $\|\varepsilon\| \leq C H\left\|\mathbf{e}_{h}\right\|_{\boldsymbol{\Lambda}}$, and (4.13) is proved.

To complete the proof for $i=0$, it only remains to estimate the second term on the right hand side of (4.12). For this we let $\mathbf{q}=\mathbf{S}\left(\mathbf{c u r l}_{H} \mathbf{z}_{H}\right)$. Since $\mathbf{q}$ is divergence free,

$$
\begin{aligned}
\left(\operatorname{grad} \phi_{h}, \operatorname{curl}_{H} \mathbf{z}_{H}\right) & =\left(\operatorname{grad} \phi_{h}, \operatorname{curl}_{H} \mathbf{z}_{H}-\mathbf{q}\right) \\
& \leq\left\|\operatorname{grad} \phi_{h}\right\|\left\|\operatorname{curl}_{H} \mathbf{z}_{H}-\mathbf{q}\right\| \\
& \leq C H\left\|\mathbf{e}_{h}\right\|\left\|\operatorname{curl} \mathbf{v}_{0}\right\|
\end{aligned}
$$


The last inequality is a consequence of $\left\|\operatorname{grad} \phi_{h}\right\| \leq\left\|\mathbf{e}_{h}\right\|$, and (4.10). This completes the proof for $i=0$.

Now fix $i \in\{1, \ldots, N\}$ and decompose $\mathbf{v}_{i}=\operatorname{grad} \psi_{i}+\operatorname{curl}_{h}^{i} \mathbf{z}_{i}$, with $\psi_{i} \in W^{i}$ and $\mathbf{z}_{i} \in \mathbf{V}^{i}$. Then, by (4.11),

$$
\left(\mathbf{u}-\mathbf{T}_{i} \mathbf{u}, \mathbf{v}_{i}\right)_{\Omega_{i}^{\prime}}=\left(\mathbf{u}-\mathbf{T}_{i} \mathbf{u}, \operatorname{curl}_{h}^{i} \mathbf{z}_{i}\right)_{\Omega_{i}^{\prime}} .
$$

The result then immediately follows from the Cauchy-Schwarz inequality and (4.1).

As a consequence of the estimate of Lemma 4.3 for $i=0$, we get that the norm of $\mathbf{I}-\mathbf{T}_{0}$ is almost bounded by one, as the following lemma shows.

Lemma 4.4. Whenever $H \leq \bar{h}_{4}$, there is a constant $c_{3}$, independent of $h$ and $H$, such that

$$
\left(1-c_{3} H\right)\left\|\mathbf{u}-\mathbf{T}_{0} \mathbf{u}\right\|_{\boldsymbol{\Lambda}}^{2} \leq\|\mathbf{u}\|_{\boldsymbol{\Lambda}}^{2} \quad \text { for all } \mathbf{u} \in \mathbf{Q}_{h} .
$$

Proof. For $i=0,1, \ldots, N$, let $\mathbf{P}_{i}: \mathbf{Q}_{h} \rightarrow \mathbf{Q}^{i}$ denote the $\boldsymbol{\Lambda}$-projection defined by

$$
\boldsymbol{\Lambda}\left(\mathbf{P}_{i} \mathbf{u}, \mathbf{v}_{i}\right)=\boldsymbol{\Lambda}\left(\mathbf{u}, \mathbf{v}_{i}\right) \text { for all } \mathbf{v}_{i} \in \mathbf{Q}^{i} .
$$

Fix $\mathbf{u}$ in $\mathbf{Q}_{h}$. We note that

$$
\begin{aligned}
\left\|\mathbf{u}-\mathbf{T}_{0} \mathbf{u}\right\|_{\boldsymbol{\Lambda}}^{2} & =\mathbf{A}\left(\mathbf{u}-\mathbf{T}_{0} \mathbf{u}, \mathbf{u}-\mathbf{P}_{0} \mathbf{u}\right)+\left(1+\omega^{2}\right)\left(\mathbf{u}-\mathbf{T}_{0} \mathbf{u}, \mathbf{u}-\mathbf{T}_{0} \mathbf{u}\right) \\
& =\boldsymbol{\Lambda}\left(\mathbf{u}, \mathbf{u}-\mathbf{P}_{0} \mathbf{u}\right)+\left(1+\omega^{2}\right)\left(\mathbf{u}-\mathbf{T}_{0} \mathbf{u}, \mathbf{P}_{0} \mathbf{u}-\mathbf{T}_{0} \mathbf{u}\right) .
\end{aligned}
$$

The result follows by applying the Cauchy-Schwarz inequality, Lemma 4.3 and noting that

$$
\begin{aligned}
\left\|\mathbf{u}-\mathbf{P}_{0} \mathbf{u}\right\|_{\boldsymbol{\Lambda}} & \leq\|\mathbf{u}\|_{\boldsymbol{\Lambda}}, \\
\left\|\mathbf{P}_{0} \mathbf{u}-\mathbf{T}_{0} \mathbf{u}\right\|_{\boldsymbol{\Lambda}} & \leq\left\|\mathbf{u}-\mathbf{T}_{0} \mathbf{u}\right\|_{\boldsymbol{\Lambda}} .
\end{aligned}
$$

Note that a consequence of Lemma 4.4 is that $\mathbf{T}_{0}$ is stable, i.e., for sufficiently small $H$,

$$
\left\|\mathbf{T}_{0} \mathbf{u}\right\|_{\Lambda} \leq C\|\mathbf{u}\|_{\Lambda}
$$

for all $\mathbf{u} \in \mathbf{Q}_{h}$.

The proofs of our main theorems depend on results for the positive operator $\boldsymbol{\Lambda}$ given in [16, 25]. There it is proved that, for any $\mathbf{u} \in \mathbf{Q}_{h}$,

$$
\begin{aligned}
C \boldsymbol{\Lambda}(\mathbf{u}, \mathbf{u}) & \leq \boldsymbol{\Lambda}\left(\sum_{i=0}^{N} \mathbf{P}_{i} \mathbf{u}, \mathbf{u}\right), \\
\sum_{i=0}^{N}\left\|\mathbf{P}_{i} \mathbf{u}\right\|_{\boldsymbol{\Lambda}, \Omega_{i}^{\prime}}^{2} & \leq(\rho+1)\|\mathbf{u}\|_{\boldsymbol{\Lambda}}^{2}
\end{aligned}
$$

We can now prove the main theorems.

Proof of Theorem 3.1. Let $\mathbf{u}$ be in $\mathbf{Q}_{h}$. The main idea of the proof of the first inequality of the theorem is to bound $\boldsymbol{\Lambda}(\mathbf{u}, \mathbf{u})$ by $\boldsymbol{\Lambda}\left(\sum_{i=0}^{N} \mathbf{T}_{i} \mathbf{u}, \mathbf{u}\right)=\boldsymbol{\Lambda}\left(\mathbf{B}_{a} \mathbf{A u}, \mathbf{u}\right)$ 
plus a perturbation term. Using (4.16) gives

$$
\begin{aligned}
C \boldsymbol{\Lambda}(\mathbf{u}, \mathbf{u}) & \leq \sum_{i=0}^{N} \boldsymbol{\Lambda}\left(\mathbf{u}, \mathbf{P}_{i} \mathbf{u}\right) \\
& =\sum_{i=0}^{N} \mathbf{A}\left(\mathbf{T}_{i} \mathbf{u}, \mathbf{P}_{i} \mathbf{u}\right)+\left(1+\omega^{2}\right)\left(\mathbf{u}, \mathbf{P}_{i} \mathbf{u}\right) \\
& =\sum_{i=0}^{N} \boldsymbol{\Lambda}\left(\mathbf{T}_{i} \mathbf{u}, \mathbf{u}\right)+\left(1+\omega^{2}\right) \sum_{i=0}^{N}\left(\mathbf{u}-\mathbf{T}_{i} \mathbf{u}, \mathbf{P}_{i} \mathbf{u}\right) .
\end{aligned}
$$

When $H<\bar{h}_{4}$, Lemma 4.3 yields

$$
\boldsymbol{\Lambda}(\mathbf{u}, \mathbf{u}) \leq C \sum_{i=0}^{N} \boldsymbol{\Lambda}\left(\mathbf{T}_{i} \mathbf{u}, \mathbf{u}\right)+C H \sum_{i=0}^{N}\left\|\mathbf{u}-\mathbf{T}_{i} \mathbf{u}\right\|_{\boldsymbol{\Lambda}, \Omega_{i}^{\prime}}\left\|\mathbf{P}_{i} \mathbf{u}\right\|_{\boldsymbol{\Lambda}, \Omega_{i}^{\prime}}
$$

Taking $H<\min \left(\bar{h}_{3}, \bar{h}_{1}\right)$, we also have by Lemma 4.2 and Lemma 4.4 that

$$
\sum_{i=0}^{N}\left\|\mathbf{u}-\mathbf{T}_{i} \mathbf{u}\right\|_{\boldsymbol{\Lambda}, \Omega_{i}^{\prime}}^{2} \leq C \sum_{i=0}^{N}\|\mathbf{u}\|_{\boldsymbol{\Lambda}, \Omega_{i}^{\prime}}^{2} \leq C\|\mathbf{u}\|_{\boldsymbol{\Lambda}}^{2}
$$

The second inequality above is a consequence of the finite covering property. Using the Cauchy-Schwarz inequality and (4.17), we get

$$
(1-C H) \boldsymbol{\Lambda}(\mathbf{u}, \mathbf{u}) \leq C \sum_{i=0}^{N} \boldsymbol{\Lambda}\left(\mathbf{T}_{i} \mathbf{u}, \mathbf{u}\right) .
$$

The first inequality of the theorem follows by taking $\bar{h}_{1}$ small enough to make the constant on the left hand side positive.

The second inequality of the theorem follows immediately from the CauchySchwarz inequality, the stability of $\mathbf{T}_{i}$, and the finite covering property. This completes the proof of the theorem

Proof of Theorem 3.2. Fix $\mathbf{u}$ in $\mathbf{Q}_{h}$ and set $\widetilde{\mathbf{u}}=\left(\mathbf{I}-\mathbf{T}_{0}\right) \mathbf{u}$. We clearly have

$$
\|\widetilde{\mathbf{u}}-\alpha \widetilde{\mathbf{T}} \widetilde{\mathbf{u}}\|_{\boldsymbol{\Lambda}}^{2}=\|\widetilde{\mathbf{u}}\|_{\boldsymbol{\Lambda}}^{2}-2 \alpha \boldsymbol{\Lambda}(\widetilde{\mathbf{u}}, \widetilde{\mathbf{T}} \widetilde{\mathbf{u}})+\alpha^{2}\|\widetilde{\mathbf{T}} \widetilde{\mathbf{u}}\|_{\boldsymbol{\Lambda}}^{2}
$$

By Lemma 4.2 and finite covering, there is a constant, say $C_{2}>0$, independent of $h$ and $H$, such that $\|\widetilde{\mathbf{T}} \widetilde{\mathbf{u}}\|_{\boldsymbol{\Lambda}}^{2} \leq C_{2}\|\widetilde{\mathbf{u}}\|_{\boldsymbol{\Lambda}}^{2}$ for $H \leq \bar{h}_{4}$. Moreover, since $\mathbf{T}_{0} \widetilde{\mathbf{u}}=0$, we have, by Theorem 3.1

$$
\boldsymbol{\Lambda}(\widetilde{\mathbf{u}}, \widetilde{\mathbf{T}} \widetilde{\mathbf{u}})=\boldsymbol{\Lambda}\left(\widetilde{\mathbf{u}}, \sum_{i=0}^{N} \mathbf{T}_{i} \widetilde{\mathbf{u}}\right) \geq c_{1} \boldsymbol{\Lambda}(\widetilde{\mathbf{u}}, \widetilde{\mathbf{u}}) .
$$

Consequently, (4.19) implies that

$$
\|\widetilde{\mathbf{u}}-\alpha \widetilde{\mathbf{T}} \widetilde{\mathbf{u}}\|_{\boldsymbol{\Lambda}}^{2} \leq\left(1-2 c_{1} \alpha+C_{2} \alpha^{2}\right)\|\widetilde{\mathbf{u}}\|_{\boldsymbol{\Lambda}}^{2} .
$$

Now if we take $\alpha_{0}=c_{1} / C_{2}$, then for any $\alpha \leq \alpha_{0}$,

$$
1-2 c_{1} \alpha+C_{2} \alpha^{2}=\tilde{\gamma}(\alpha) \equiv \tilde{\gamma}
$$

is less than 1. Combining this with Lemma 4.4, we get that when $H \leq \bar{h}_{4}$ and $1-c_{3} H>0$,

$$
\left\|(\mathbf{I}-\alpha \widetilde{\mathbf{T}})\left(\mathbf{I}-\mathbf{T}_{0}\right) \mathbf{u}\right\|_{\Lambda}^{2} \leq \frac{\widetilde{\gamma}}{1-c_{3} H}\|\mathbf{u}\|_{\Lambda}^{2} .
$$


Clearly there is an $\bar{h}_{2}(\alpha)=\bar{h}_{2}>0$ such that $\widetilde{\gamma}\left(1-c_{3} \bar{h}_{2}\right)^{-1}=1-(1-\widetilde{\gamma}) / 2 \equiv \gamma$. This completes the proof of the theorem.

The estimate of Theorem 3.2 implies that $\mathbf{B}_{m}$ can be used to precondition a linear iterative method. Indeed, the iterates $\mathbf{v}_{i}, i=1,2, \ldots$, defined by

$$
\mathbf{v}_{i}=\mathbf{v}_{i-1}+\mathbf{B}_{m}\left(\ell-\mathbf{A} \mathbf{v}_{i-1}\right), \quad i=1,2, \ldots,
$$

with some initial guess $\mathbf{v}_{0}$, converge to the solution of the linear system $\mathbf{A v}=\ell$, i.e.,

$$
\left\|\mathbf{v}-\mathbf{v}_{i}\right\|_{\boldsymbol{\Lambda}} \leq \gamma^{i}\left\|\mathbf{v}-\mathbf{v}_{0}\right\|_{\boldsymbol{\Lambda}}
$$

Alternatively, Theorem 3.2 can be used to obtain bounds for $\mathbf{B}_{m} \mathbf{A}$ analogous to those of Theorem 3.1 Indeed, when $H$ is sufficiently small, there exist positive constants $c_{4}$ and $c_{5}$, independent of $h$ and $H$, such that, for all $\mathbf{u} \in \mathbf{Q}_{h}$,

$$
\begin{gathered}
c_{4} \boldsymbol{\Lambda}(\mathbf{u}, \mathbf{u}) \leq \boldsymbol{\Lambda}\left(\mathbf{B}_{m} \mathbf{A} \mathbf{u}, \mathbf{u}\right) \\
\boldsymbol{\Lambda}\left(\mathbf{B}_{m} \mathbf{A} \mathbf{u}, \mathbf{v}\right) \leq c_{5} \boldsymbol{\Lambda}(\mathbf{u}, \mathbf{u})^{1 / 2} \boldsymbol{\Lambda}(\mathbf{v}, \mathbf{v})^{1 / 2}
\end{gathered}
$$

The first inequality follows directly from Theorem 3.2 with $c_{4}=\left(1-\gamma^{1 / 2}\right)$, since

$$
\boldsymbol{\Lambda}\left(\left(\mathbf{I}-\mathbf{B}_{m} \mathbf{A}\right) \mathbf{u}, \mathbf{u}\right) \leq \gamma^{1 / 2} \boldsymbol{\Lambda}(\mathbf{u}, \mathbf{u})
$$

The second inequality above follows from the identity

$$
\mathbf{B}_{m} \mathbf{A}=\alpha \widetilde{\mathbf{T}}+\mathbf{T}_{0}-\alpha \widetilde{\mathbf{T}} \mathbf{T}_{0}
$$

the stability of $\mathbf{T}_{i}$, and the finite covering property. Thus $\mathbf{B}_{m}$ is also a good preconditioner for use in GMRES.

\section{Numerical Results}

In this section we report results of numerical experiments confirming and illustrating the theory in the previous sections. All of the computations to be described use lowest order Nedelec elements on cubes. The domain $\Omega$ is taken to be the unit cube $(0,1)^{3}$ and is meshed uniformly by cubic elements of size $h$. The coarse mesh is also uniform and is made up of cubes of length $H$. Overlapping subdomains are constructed by adjoining just enough fine elements to the coarse elements so that (2.5) holds with $\delta=1 / 10$.

Equation (3.4) was solved using GMRES in the inner product induced by the matrix $\left\{\Lambda_{i j}\right\}$. The right hand side $b$ of (3.4) was chosen such that the solution $u$ is the vector of nodal coefficients of the interpolant of

$$
[y(1-y) z(1-z), y x(1-x) z(1-z), x(1-x) y(1-y)] \text {. }
$$

In all reported computations, GMRES was iterated until the norm of the residual was reduced by a factor of $10^{-6}$, restarting after every 50 iterations. Iteration counts are reported in Table 5.1 for the case $\omega=1$. They appear bounded, as predicted by Corollary 3.1. Note that Corollary 3.1] guarantees that GMRES if restarted after each iteration would still yield bounded iteration counts. Although we do not report the iteration counts for this case, in our experiments this was indeed the case.

Results obtained using the multiplicative preconditioner with $\alpha=1 / 4$ are given in Table 5.2 Here $B_{m}$ denotes the matrix corresponding to the operator $\mathbf{B}_{m}$ defined analogously to $B_{a}$. The multiplicative preconditioner appears to perform slightly better than the additive. 
TABLE 5.1. GMRES iteration counts with $B_{a}$ and $\omega=1$.

\begin{tabular}{cc|ccccc}
\multicolumn{1}{c}{} & \multicolumn{6}{c}{$H$} \\
& & $1 / 2$ & $1 / 4$ & $1 / 8$ & $1 / 16$ & $1 / 32$ \\
\hline \multirow{4}{*}{$h \quad 1 / 4$} & 13 & - & - & - & - \\
& $1 / 8$ & 16 & 18 & - & - & - \\
& $1 / 16$ & 20 & 19 & 18 & - & - \\
& $1 / 32$ & 20 & 21 & 17 & 17 & - \\
& $1 / 64$ & 21 & 22 & 20 & 16 & 16
\end{tabular}

TABLE 5.2. GMRES iteration counts with $B_{m}$ and $\omega=1$.

\begin{tabular}{cc|ccccc} 
& & \multicolumn{6}{c}{$H$} \\
\multirow{4}{*}{$h$} & & $1 / 2$ & $1 / 4$ & $1 / 8$ & $1 / 16$ & $1 / 32$ \\
\hline & $1 / 4$ & 12 & - & - & - & - \\
& $1 / 8$ & 14 & 15 & - & - & - \\
& $1 / 16$ & 17 & 16 & 14 & - & - \\
& $1 / 32$ & 18 & 18 & 15 & 12 & - \\
& $1 / 64$ & 18 & 19 & 17 & 13 & 12
\end{tabular}

TABLE 5.3. GMRES iteration counts with $B_{a}$ and $\omega=10$.

\begin{tabular}{cc|ccccc}
\multicolumn{1}{c}{} & \multicolumn{6}{c}{$H$} \\
& & $1 / 2$ & $1 / 4$ & $1 / 8$ & $1 / 16$ & $1 / 32$ \\
\hline \multirow{4}{*}{$h$} & $1 / 4$ & 20 & - & - & - & - \\
$1 / 8$ & 23 & 44 & - & - & - \\
& $1 / 16$ & 46 & 237 & 98 & - & - \\
& $1 / 32$ & 53 & 106 & 32 & 24 & - \\
& $1 / 64$ & 54 & 118 & 33 & 21 & 18
\end{tabular}

TABLE 5.4. GMRES iteration counts with $B_{m}$ and $\omega=10$.

\begin{tabular}{cc|ccccc} 
& & \multicolumn{6}{c}{$H$} \\
& & $1 / 2$ & $1 / 4$ & $1 / 8$ & $1 / 16$ & $1 / 32$ \\
\hline \multirow{4}{*}{$h$} & $1 / 4$ & 19 & - & - & - & - \\
& $1 / 8$ & 23 & 38 & - & - & - \\
& $1 / 16$ & 82 & 48 & 17 & - & - \\
& $1 / 32$ & 99 & 51 & 21 & 14 & - \\
& $1 / 64$ & 98 & 50 & 24 & 16 & 12
\end{tabular}

The restriction that $H$ should be small enough in our theorems is not merely an artifact of the theory. Although iteration counts were uniformly good for all $H$ in Tables 5.1 and 5.2, this is no longer the case for large $\omega$. To illustrate this, we report iteration counts for the case $\omega=10$ in Tables 5.3 and 5.4. Clearly in this case the coarse grid needs to be fine enough for a good preconditioner to result. Heuristic arguments indicating that the coarse mesh size $H$ should not be taken larger than $\pi / \omega$ exist in the literature [3]. Tables 5.3 and 5.4 indicate that taking $H=\pi / \omega$ may not be sufficient for lowest order elements.

Finally we investigate if we can replace the subdomain inversions by an alternate operation on subdomains and still get a good preconditioner as in elliptic problems. 
TABLE 5.5. GMRES iteration counts with $B_{a}^{\times}$and $\omega=1$. (An entry $n^{+}$indicates that the convergence criterion was not met even after $n$ iterations.)

\begin{tabular}{cc|ccccc} 
& & \multicolumn{5}{c}{$H$} \\
\multirow{4}{*}{$h$} & & $1 / 2$ & $1 / 4$ & $1 / 8$ & $1 / 16$ & $1 / 32$ \\
\hline \multirow{4}{*}{$1 / 4$} & 23 & - & - & - & - \\
& $1 / 8$ & 52 & 131 & - & - & - \\
& $1 / 16$ & 73 & 212 & 645 & - & - \\
& $1 / 32$ & 101 & 119 & 766 & $1000^{+}$ & - \\
& $1 / 64$ & 111 & 127 & 107 & 271 & $1000^{+}$
\end{tabular}

TABLE 5.6. GMRES iteration counts with $B_{m}^{\times}$and $\omega=1$.

\begin{tabular}{cc|ccccc} 
& & \multicolumn{6}{c}{$H$} \\
& & $1 / 2$ & $1 / 4$ & $1 / 8$ & $1 / 16$ & $1 / 32$ \\
\hline \multirow{4}{*}{$h$} & $1 / 4$ & 22 & - & - & - & - \\
& $1 / 8$ & 39 & 89 & - & - & - \\
& $1 / 16$ & 63 & 71 & 163 & - & - \\
& $1 / 32$ & 86 & 97 & 69 & 92 & - \\
& $1 / 64$ & 92 & 106 & 94 & 62 & 169
\end{tabular}

Let $B_{a}^{\times}$be the matrix of the operator $\mathbf{B}_{a}^{\times}$defined by

$$
\mathbf{B}_{a}^{\times}(\ell)=\sum_{i=0}^{N} \mathbf{v}_{i},
$$

where $\mathbf{v}_{0}$ solves

$$
\mathbf{A}\left(\mathbf{v}_{0}, \mathbf{w}_{0}\right)=\ell\left(\mathbf{w}_{0}\right) \quad \text { for all } \mathbf{w}_{0} \in \mathbf{Q}^{0},
$$

while the $\mathbf{v}_{i}$ for $i=1, \ldots, N$ satisfy

$$
\mathbf{\Lambda}\left(\mathbf{v}_{i}, \mathbf{w}_{i}\right)=\ell\left(\mathbf{w}_{i}\right) \quad \text { for all } \mathbf{w}_{i} \in \mathbf{Q}^{i} .
$$

The results obtained using $B_{a}^{\times}$as preconditioner in GMRES are shown in Table 5.5 and these suggest that $B_{a}^{\times}$is not a good preconditioner (see also Remark 3.2). Similar results are obtained (see Table 5.6) for $B_{m}^{\times}$defined by multiplicatively combining the coarse and local solution operators of (5.1) and (5.2) as in the definition of $B_{m}$.

\section{REFERENCES}

1. C. Amrouche, C. Bernardi, M. Dauge, and V. Girault, Vector potentials in three-dimensional nonsmooth domains, Math. Methods Appl. Sci. 21 (1998), no. 9, 823-864. MR 99e:35037

2. Douglas N. Arnold, Richard S. Falk, and Ragnar Winther, Multigrid in $\mathbf{H}(\operatorname{div})$ and $\mathbf{H}(\mathbf{c u r l})$, Numer. Math. 85 (2000), no. 2, 197-217. MR 2001d:65161

3. Rudolf Beck, Peter Deuflhard, Ralf Hiptmair, Ronald H. W. Hoppe, and Barbara Wohlmuth, Adaptive multilevel methods for edge element discretizations of Maxwell's equations, Surveys Math. Indust. 8 (1999), 271-312. MR 2000i:65206

4. Alain Bossavit, A rationale for "edge-elements" in 3-D fields computations, IEEE Trans. Mag. 24 (1988), no. 1, 74-79.

5. Franco Brezzi and Michel Fortin, Mixed and Hybrid Finite Element Methods, Springer Series in Computational Mathematics, no. 15, Springer-Verlag, New York, 1991. MR 92d:65187

6. Xiao-Chuan Cai and Olof B. Widlund, Domain decomposition algorithms for indefinite elliptic problems, SIAM J. Sci. Stat. Comput. 13 (1992), no. 1, 243-258. MR 92i:65181

7. L. Demkowicz and L. Vardapetyan, Modeling of electromagnetic absorption/scattering problems using hp-adaptive finite elements, Comput. Methods Appl. Mech. Engrg. 152 (1998), 103-124. MR 99b:78003 
8. M. Dryja and O. B. Widlund, An additive variant of the Schwarz alternating method for the case of many subregions, Tech. Report 339, Courant Institute of Mathematical Sciences, New York, 1987.

9. Large Linear Systems, Academic Press, San Diego, 1989, Proceedings of a conference held at Austin, Texas, in October 1988, pp. 273-291. MR 91f:65071

10. _ Towards a unified theory of domain decomposition algorithms for elliptic problems, Third International Symposium on Domain Decomposition Methods for Partial Differential Equations, SIAM, Philadelphia, 1990, Symposium held at Houston, Texas, in March 1989, 3-21. MR 91m:65294

11. Stanley C. Eisenstat, Howard C. Elman, and Martin H. Schultz, Variational iterative methods for nonsymmetric systems of linear equations, SIAM J. Numer. Anal. 20 (1983), no. 2, 345357. MR 84h:65030

12. V. Girault, Incompressible finite element methods for Navier-Stokes equations with nonstandard boundary conditions in $\mathbf{R}^{3}$, Math. Comp. 51 (1988), no. 183, 55-74. MR 90e:65155

13. Vivette Girault and Pierre-Arnaud Raviart, Finite Element Methods for Navier-Stokes Equations, Springer series in Computational Mathematics, no. 5, Springer-Verlag, New York, 1986. MR 88b:65129

14. Jayadeep Gopalakrishnan, Joseph E. Pasciak, and Leszek Demkowicz, A multigrid algorithm for time harmonic Maxwell equations, In preparation.

15. Ralf Hiptmair, Multigrid method for Maxwell's equations, SIAM J. Numer. Anal. 36 (1999), no. 1, 204-225. MR 99j:65229

16. Ralf Hiptmair and Andrea Toselli, Overlapping Schwarz methods for vector valued elliptic problems in three dimensions, Parallel solution of PDEs, IMA Volumes in Mathematics and its Applications, Springer-Verlag, Berlin, 1998.

17. R. Leis, Exterior boundary-value problems in mathematical physics, Trends in Applications of Pure Mathematics to Mechanics, Volume II (Henryk Zorski, ed.), Monographs and Studies in Mathematics, no. 5, Pitman, London, 1979, A collection of papers presented at a symposium at Kozubnik, Poland, in September 1977, pp. 187-203. MR 81d:78016

18. P. L. Lions, On the Schwarz alternating method, First International Symposium on Domain Decomposition Methods for Partial Differential Equations (Roland Glowinski, Gene H. Golub, Gérard A. Meurant, and Jacques Périaux, eds.), SIAM, Philadelphia, 1988, Symposium held at Ecole Nationale des Ponts et Chaussées, Paris, in January 1987, pp. 1-42. MR 90a:65248

19. P. Monk and L. Demkowicz, Discrete compactness and the approximation of Maxwell's equations in $\mathbf{R}^{3}$, Math. Comp. 70 (2001), 507-523. MR 2001g:65156

20. Peter Monk, A finite element method for aproximating the time-harmonic Maxwell equations, Numer. Math. 63 (1992), 243-261. MR 94b:65134

21. J. C. Nedelec, Mixed Finite Elements in $\mathbb{R}^{3}$, Numer. Math. 35 (1980), 315-341. MR 81k:65125

22. Waldemar Rachowicz, Leszek Demkowicz, Andrzej Bajer, and Timothy Walsh, A two-grid iterative solver for stationary Maxwell's equations, Iterative Methods in Scientific Computation II (D. Kincaid et al., eds.), IMACS, 1999.

23. Y. Saad and M. H. Schultz, GMRES: A generalized minimal residual algorithm for solving nonsymmetric linear systems, SIAM J. Sci. Statist. Comput. 7 (1986), 856-869. MR 87g:65064

24. Barry F. Smith, Petter E. Bjørstad, and William D. Gropp, Domain Decomposition. Parallel Multilevel Methods for Elliptic Partial Differential Equations, Cambridge University Press, Cambridge, 1996. MR 98g:65003

25. A. Toselli, Overlapping Schwarz methods for Maxwell's equations in three dimensions, Numer. Math. 86 (2000), 733-752. MR 2001h:65137

26. Andrea Toselli, Olof B. Widlund, and Barbara I. Wohlmuth, An iterative substructuring method for Maxwell's equations in two dimensions, Math. Comp. 70 (2000), 935-949. MR 2001j:65140

Institute for Mathematics and its Applications, Minneapolis, Minnesota 55455

E-mail address: jayg@ima.umn.edu

Texas A\&M University, College Station, Texas 77843-3368.

E-mail address: pasciak@math.tamu.edu 